\title{
Methodology for measuring geometrical and thermal characteristics of flames in laboratory forest fires
}

\author{
E. Pastor, A. Àgueda, L. Martin, M. Muñoz, A. León, Y. Pérez \\ \& E. Planas \\ Centre d'Estudis del Risc Tecnolòic (CERTEC), \\ Department of Chemical Engineering, \\ Universitat Politècnica de Catalunya (UPC), Spain
}

\begin{abstract}
Flame properties from forest fuels are needed for fire behaviour modelling, either for developing empirical models or for validation purposes. Measuring such properties is usually a very complex task due to the fluctuation in time and space and to the ambiguity in the definition of several geometrical variables. Moreover, there is a lot of subjectivity in most of the methods historically used in fire laboratories, which should be strongly avoided. In this paper, we present a standard, objective and automated methodology to determine several geometrical and thermal properties of laboratory and open stationary fires, based on IR and visible imagery techniques. We develop several procedures to obtain the mean flame height based on the concept of flame intermittence, the flame pulse frequency, the temperatures distribution within the flame and the emissive power and the radiation flux of the flames.
\end{abstract}

Keywords: flame geometry, thermal characteristics, IR imagery, stationary fires.

\section{Introduction}

Understanding flame properties and behavior is an essential issue in predicting forest fire spread and suppression potential. Both geometrical and thermal flame characteristics are needed as input variables in different sorts of fire studies. Flame length and angle are required for radiative heat transfer calculations [1], crowning potential modeling [2] and suppression difficulty estimations [3]. 
Knowing potential flame dimensions and thermal properties are important to assess fuel-breaks efficiency [4]. Also, flame structure has to be very well described to validate the new generation of fire behaviour CFD-type prediction models that are being developed so far [5].

Obtaining accurate flames data is a very complex task, not only because of the transient nature of flames, but also due in one hand to the lack of common definitions and procedures, and on the other due to the subjective methodologies that are usually applied, which are mainly based on visual estimations. There is certainly a need of defining and using standards, as stated in Anderson et al. [6]. In terms of methods to be used, the authors strongly suggest objective measurement procedures. Visual estimations to determine flame dimensions might have to be done at large-scale field experiments but should be strongly avoided in laboratory fires. Information obtained from small or medium scale laboratory experiments, either when simulating moving or stationary fires, has to be as accurate and complete as possible, since these tests represent the controlled scenario to study some complex aspects of forest fires. So, rather than to make punctual lectures, under controlled conditions automated procedures providing a high quality dataset in terms of frequency and cover of the measure should be used. Again, there is a lack of standard in objective computerized procedures, since the variability in time of flame characteristics has been commonly considered following different criteria. For instance, flame height which is strongly related to the flame pulsating phenomenon, has been generally averaged using imprecise methods -excepting a few examples [7]-, like analysing a small sample of frames with a frequency lower than the flame pulse [8], or averaging a certain number of randomly selected peaks at the maximum flame height [9]. This might induce big errors when studying much variable flames. Concerning technology to obtain thermal flame properties, point measurements using thermocouples and heat flux sensors can give a very reliable and dense dataset but they can cover only points or narrow areas of the flame, so combining this technology with IR imagery is highly desirable.

In this paper, we present an objective and automated methodology, developed basically with MATLAB ${ }^{\circledR}$ algorithms, to determine several geometrical and thermal properties of controlled stationary fires (laboratory and open) based on IR and visible imagery techniques. We calculate flame height overcoming the problem of data averaging using Zukoski's definition of flame intermittency [10] and obtaining flame pulse frequency. We study the temperature evolution over time and throughout the flame and we compute the emissive power and the radiant heat flux of the flames.

\section{Experimental methods}

\subsection{Laboratory setup and tests}

The laboratory tests were performed in a combustion chamber at CERTEC's fire laboratory (FlamesLab), fig. 1. 


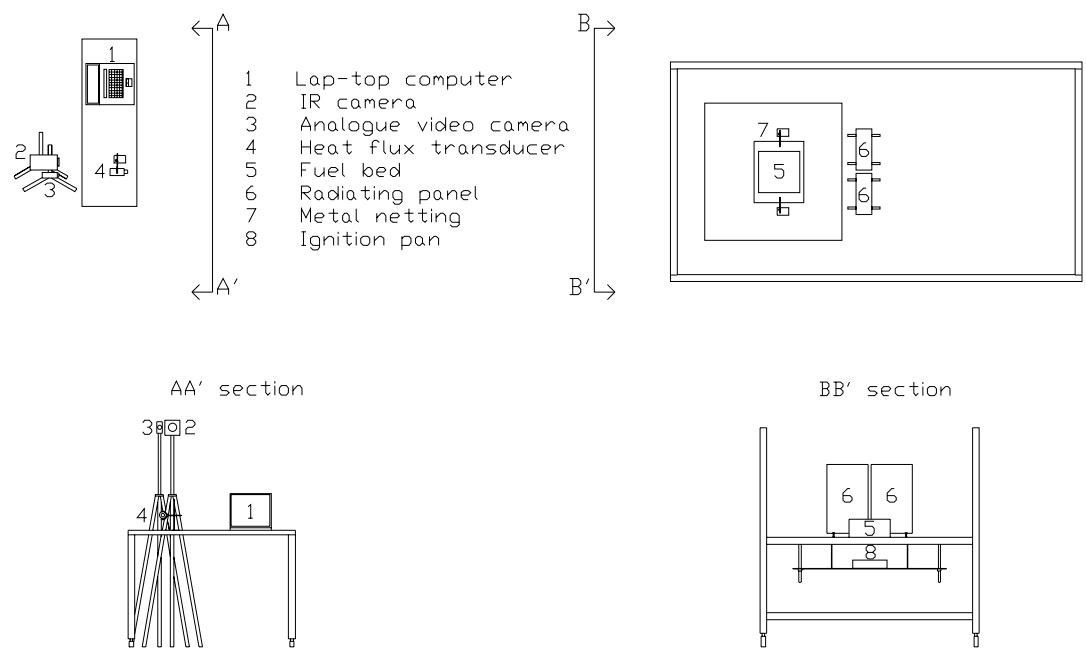

Figure 1: Experimental layout.

The forest fuel is placed on top of a metal netting $(45 \mathrm{~cm} \mathrm{x} 40 \mathrm{~cm})$ which allows ignition to take place underneath. Once ignited, the test is monitored by different sensors located at $4.75 \mathrm{~m}$ in front of the fuel bed, obtaining the suitable view of the whole flame. The equipment set up is composed by an IR camera (AGEMA Thermovision 570-Pro) operating at the TIR window, with an available range of brightness temperature of $-20^{\circ} \mathrm{C}$ to $1500^{\circ} \mathrm{C}$ and a $5 \mathrm{~Hz}$ recording frequency of $240 \times 320$ pixels frames; 1 heat flux transducer (64-2-16 MEDTHERM, thermopile Schimdt-Boelter) with a range of $0-23 \mathrm{~kW} / \mathrm{m}^{2}$ and an analogue video camera (SONY Handycam Vision CCD-TR840E) working at $25 \mathrm{~Hz}$. All the sensors but the video camera are controlled and triggered by a laptop computer using tailor-made software that allows synchronising all the data readings. Behind the fuel bed, two $30 \mathrm{~cm}$ x $50 \mathrm{~cm}$ surface radiating panels are positioned at both sides of the axis of symmetry of the bed, one next to the other. These panels act either as hot black bodies when they are on (with temperatures sets of $300^{\circ} \mathrm{C}$ and $400^{\circ} \mathrm{C}$ ) and as geometric references for image analysis.

The tests used to design this methodology were performed using fuel beds made of Pinus halepensis needles; oven dried $\left(80^{\circ} \mathrm{C}, 24 \mathrm{~h}\right)$ and arranged as parallelepipeds $(200 \times 200 \times 155 \mathrm{~mm})$ with a bulk density of $30 \mathrm{~kg} / \mathrm{m}^{3}$. The ambient conditions were around $22^{\circ} \mathrm{C}$ and $45 \%$ of relative humidity. The ignition source used was a plate full of sand wet of alcohol that provided homogeneous pilot flames.

Four different phases in terms of flame's behaviour could be easily distinguished for each test. An ignition phase of around 25-30 s; following, a pseudo-stationary combustion lasting again around $25 \mathrm{~s}$ in which all the flame's studies were performed, a third phase of transient combustion showing turbulent 
and erratic flame behaviour for about 10-15 s, and finally an extinguishing phase characterized by glowing combustion of the remaining fuel residue.

\subsection{Flame height and flame pulse frequency}

Flame height evolution with time was obtained for several tests, using the video sequence recorded during the pseudo-stationary phase. A flame height value was automatically calculated for each single frame by means of an algorithm which had as inputs the luminosity threshold for the flame, the location of the flame base and the pixel dimensions.

The threshold value for luminosity was determined by transforming the original RGB (Red-Green-Blue) sequence, using the HSB (Hue-SaturationBrightness) model considering only values of brightness, i.e. eliminating hue and saturation variables. The first images of both sequences (original and transformed) were then overlapped and the threshold was determined as the one by which the flame contour of both images was coincident. The base of the flame was considered at the upper edge of the fuel bed. As fuel was being consumed, this level was also descending with time, so to make the procedure easier and fully automated, a mean constant position of the flame base at $3 / 4$ of the fuel bed height was assumed. Finally, the dimensions of the pixels were calculated using known standard distances present at the background of the images.

The algorithm first output was a vector containing the flame height for each image. This was obtained computing the number of pixels from the base of the flame to the tip, considered the later as the first pixel from the top of the image where the luminosity threshold was reached. Then, given flame intermittency as the fraction of the time during which flame height is greater than a certain value [10], the algorithm calculated the minimum, the mean and the maximum flame height considering the values where flame intermittency was $0.95,0.5$ and 0.05 respectively.

The last part of the algorithm allowed us to obtain the flame pulse [11]. The first step for that was taking several periods of time within the pseudo-stationary phase of a test, where flame height was oscillating around a roughly constant value. Then for each period of time selected, we smoothed the flame height curve using a Fast Fourier transformation function (FFT), calculated the smoothed mean flame height and assured that the filtered height values oscillate around the calculated mean. The flame frequency for each period of time was computed as the number of peaks present at the smoothed curve divided by the duration of the period. Finally, an averaged frequency was obtained considering all the periods in which a test was divided.

\subsection{Temperatures distribution}

IR images were treated to study temperatures variability, first over time developing a MATLAB ${ }^{\circledR}$ algorithm and then over space (i.e. within the flame), using the ThermaCAM ${ }^{\mathrm{TM}}$ Researcher 2001 software of IR imagery analysis. Several assumptions were made for developing this methodology. Flame emissivity was considered constant throughout the flame and over time and equal 
to 0.5 . Minimum flame temperature was assumed to tally with the flame contour temperature which was considered constant along the flame perimeter.

Flame contour temperature was obtained for each test from overlapping IR and VHS images of the pseudo-stationary phase. So, IR images were scaled and aligned to VHS images in order to have the same pixel dimension and the same centre of coordinates. A working area containing the whole flame just above the fuel bed was defined and extracted, as IR and VHS images did not cover the same field of view and fuel bed temperatures were not target of analysis. After that, both sequences had to be synchronized since they had different recording frequencies. The contour temperature was determined for each IR image overlapped, as the one whose isotherm was coincident to the flames' perimeter. Then, the values of maximum flame temperature and mean flame temperature over time were extracted transforming the IR sequence of the working area to a series of flame temperature matrices, $T_{f_{(i, j) k}}$, being $(i, j)$ the pixels coordinates and $k$ the number of the image. From that, mean values were also calculated.

Temperature distribution within the flame (along the height and across the width) was studied analysing the IR images of those instants when the FFT function showed flame height peaks. The contour temperature was used as threshold value to distinguish the flame from its surroundings in the IR images. The ThermaCAM ${ }^{\mathrm{TM}}$ Researcher 2001 analysis toolbox was used to obtain the temperatures along the axis of symmetry of the flame and across the width of the flame at three different levels; the first level ( $L O 1)$ was located at the position were the flame axis had the maximum temperature and the second (LO2) and the third level (LO3) were fixed at 0.5 and 0.75 of the maximum height, respectively.

\subsection{Emissive power, radiation heat flux and flame emissivity}

Flame emissivity, emissive power of the flame and radiation heat flux of the flame captured by the transducer was calculated combining the data captured by both the transducer and the IR camera. The radiation heat flux captured by the transducer was considered to be coming from two different sources, i.e. flame and fuel bed. The solid body model was selected to calculate both fluxes and emissive power was expressed using Stefan-Boltzmann's law. Then, the following equation applied:

$$
\varepsilon_{f} \cdot \sigma \cdot\left[T_{f_{(i, j) k}}\left(\varepsilon_{f}\right)\right]^{4} \cdot \tau \cdot F_{f}=Q_{r_{t}}-\varepsilon_{b} \cdot \sigma \cdot T_{b_{(i, j) k}}^{4} \cdot \tau \cdot F_{b}
$$

where:

$\begin{array}{ll}\varepsilon_{f} & \text { Flame emissivity (-) } \\ \sigma & \text { Stefan-Boltzmann constant }\left(5.67 \cdot 10^{-8} \mathrm{~W} \cdot \mathrm{m}^{-2} \cdot \mathrm{K}^{-4}\right) \\ \tau & \text { Atmospheric transmissivity (-) } \\ F_{f} & \text { View factor of the flame (-) } \\ Q_{r_{t}} & \text { Radiation heat flux received by the transducer }\left(\mathrm{kW} / \mathrm{m}^{2}\right) \\ \varepsilon_{b} & \text { Fuel bed emissivity (-) }\end{array}$


$T_{f_{(i, j) k}}\left(\varepsilon_{f}\right)$ Flame temperature as a function of emissivity (K)

$T_{b_{(i, j) k}} \quad$ Fuel bed temperature (K)

$F_{b} \quad$ View factor of the fuel bed (-)

The atmospheric transmissivity was determined using the specific model integrated in the ThermaCAM ${ }^{\mathrm{TM}}$ Researcher 2001. Fuel bed was assumed behaving as a black body with emissivity equal to 1 and fuel bed temperature matrices were extracted from the IR sequences considering only the area occupied by the fuel bed. View factors were calculated assuming a finite emitting area composed by a summation of pixels (the flame and the fuel bed respectively) and a differential element as a receptor (the transducer). Flame temperature was considered as a function of flame emissivity, and thus an iterative method was used to solve eqn. (1).

\subsection{Field experiments}

The methodology developed was validated at a larger scale with bigger stationary fires in the open (fig. 2). Temperature distribution of the flame could not be extracted from IR imagery, since the flame radiation captured by the IR camera was disrupted by the radiation emitted by the radiating panels placed just behind. However, as temperature of the panels was known, emissive power and radiation heat flux received by the sensor that was coming from the panels could be calculated, and thus a mean value for flame temperature could be obtained using eqn. (1).

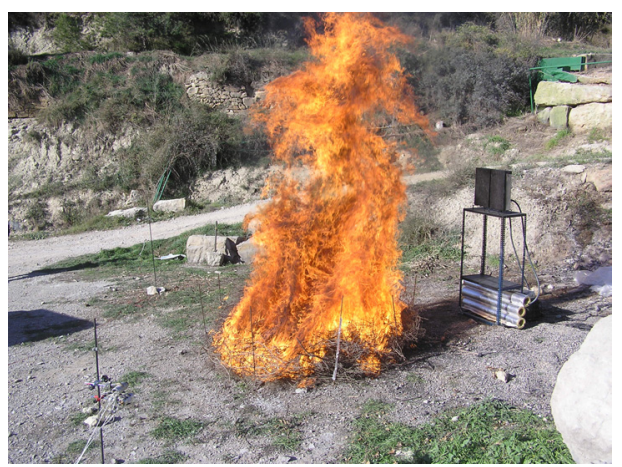

Figure 2: Open stationary fires.

The tests were performed at the CERTEC's premises at Can Padró fire testing field. We burned circular piles of $2.5 \mathrm{~m}$ diameter and around $0.7 \mathrm{~m}$ height, made of $40 \mathrm{~kg}$ of forest fuel less than $15 \mathrm{~mm}$. The fuel was previously collected and transported from a site close to the experimental area where some fuel reduction treatments were applied before. We tested 4 different Mediterranean species at around $15 \%$ of fuel moisture under $10^{\circ} \mathrm{C}$ of 
atmospheric temperature and $70 \%$ of relative humidity. The same devices were used and placed the same way as in the laboratory tests, taking into account to monitor the whole area occupied by the flame. Ignition was homogeneously made covering the entire fuel bed surface by means of two drip torches. Again, several combustion phases were identified; the ignition lasted around 50 seconds; then it followed a 10 s phase of flame growing; after, a pseudostationary phase was considered as the entire period where the flame was fully developed and lasted around $30 \mathrm{~s}$; then a much turbulent and erratic flame showed a transient combustion phase of around 30 seconds and finally, and finally an extinction phase of around 2 min characterized by a slow combustion of the remaining fuel was observed.

\section{Results and discussion}

The results derived from applying the methodology developed are presented using two tests as examples, one from the laboratory and the other from the field corresponding to one of the experiments at the open performed with Quercus ilex. Fig. 3 shows the two steps outcome of the algorithm designed to obtain flame height. For the lab test, considering an equivalent fuel bed diameter $(\Phi)$ of $225 \mathrm{~mm}$, the height giving 0.5 flame intermittency, i.e. representing the mean flame height, equals $880 \mathrm{~mm}$, whereas for the field test this height is $1250 \mathrm{~mm}$. Experimental values are compared to one of the tests collected in [10], made with gaseous fuels on a circular burner.
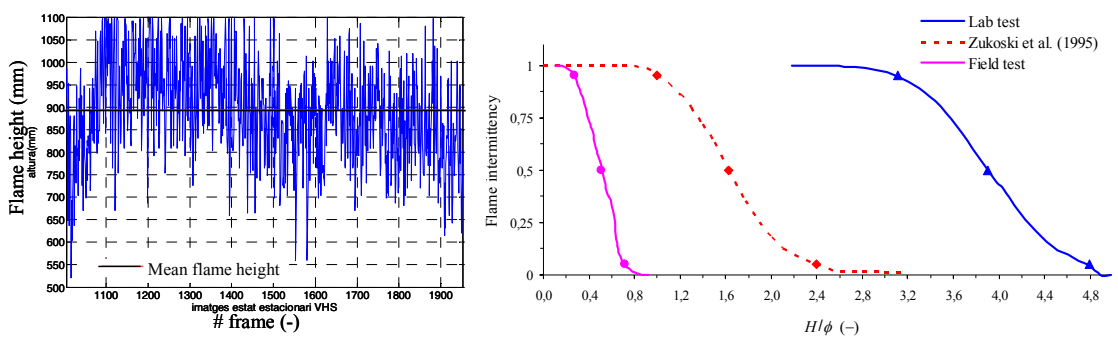

Figure 3: Flame height evolution during the pseudo-stationary phase for the lab test (left). Flame intermittency versus characteristic dimension for lab and field tests (right).

The intermittency study can also provide flame height values for the continuous flame region (0.95 intermittency, i.e. minimum flame height) and maximum flame height values which correspond to an intermittency of 0.05 ; being $750 \mathrm{~mm}$ and $1750 \mathrm{~mm}$ for the field test, and $699 \mathrm{~mm}$ and $1083 \mathrm{~mm}$ for the lab test, respectively. It can be extracted from these values that the intermittent region is larger for the field test than for the lab test, since the continuous flame region occupies the $43 \%$ of the maximum flame height for the field test, whereas it corresponds to the $65 \%$ of the maximum flame height at the laboratory test. Fig. 4 shows the two steps outcome of the algorithm designed to obtain flame 
pulse frequency. The two values found for lab $(3.10 \mathrm{~Hz})$ and field tests $(1.07 \mathrm{~Hz})$ are in agreement with the Pagni [12] function for flame frequency developed for different fuels and diameters.
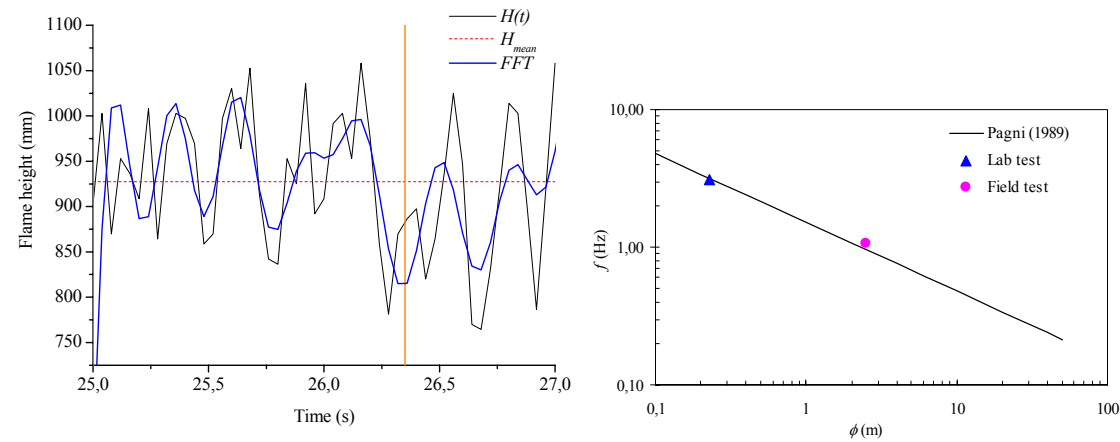

Figure 4: $\quad$ Flame pulse analysis for one period of the pseudo-stationary phase for the lab test (left). Flame pulse $(f)$ versus fuel bed diameter $(\Phi)$ for lab and field tests (right).

The functions of maximum flame temperature and mean flame temperature over time extracted from the IR images of the lab test gave $767 \mathrm{~K}$ and $479 \mathrm{~K}$, for maximum and mean flame temperatures, respectively. The temperature distribution analysis for the lab test is shown in fig. 5. It illustrates the result from the overlapping algorithm from which a contour temperature can be extracted (left) and the position of the lines along the axis and across the flame's width (right) where the analysis was performed.
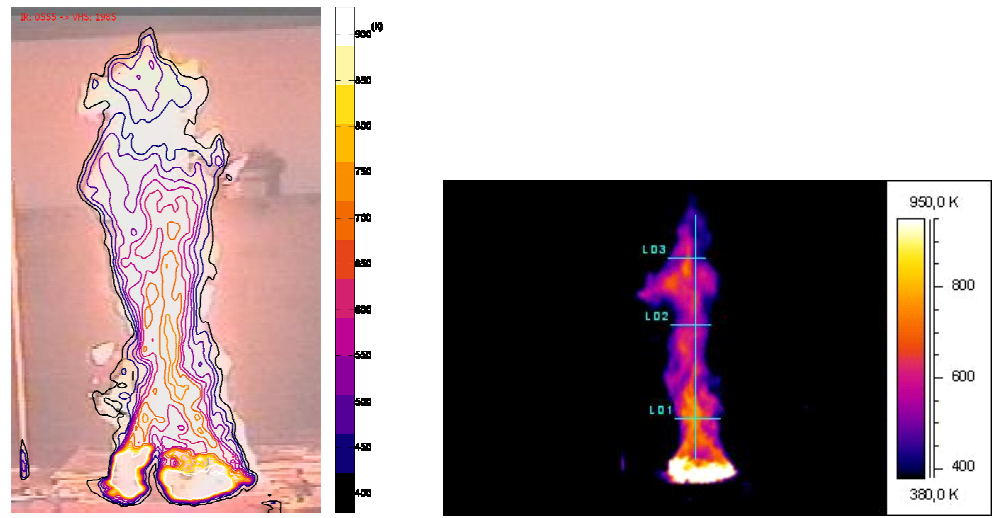

Figure 5: IR isotherms and VHS images overlapped (left). Flame temperature distribution analysis (right).

Averaged results from the lab test (fig. 6) show the dimensionless temperature -defined as the temperature of a certain pixel $T$ divided by the maximum 
temperature $T_{\max }$ of the line- along the dimensionless flame height (left) and across the dimensionless flame width (right), i.e. the position along the axis $H$ and across the width $W$ divided by the maximum flame height $H_{\max }$ and flame width $W_{\max }$, respectively. The temperature profile along the flame height shows a maximum value at around $1 / 5$ of the total flame height, and then decreases with a mean linear gradient of around $3^{\circ} \mathrm{C}$ each $10 \mathrm{~mm}$. The temperature profile with the flame width follows a similar behaviour across the three height levels analysed. The maximum temperature value is located at the axis of the flame and it decreases with a linear gradient of around $30^{\circ} \mathrm{C}$ each $10 \mathrm{~mm}$.
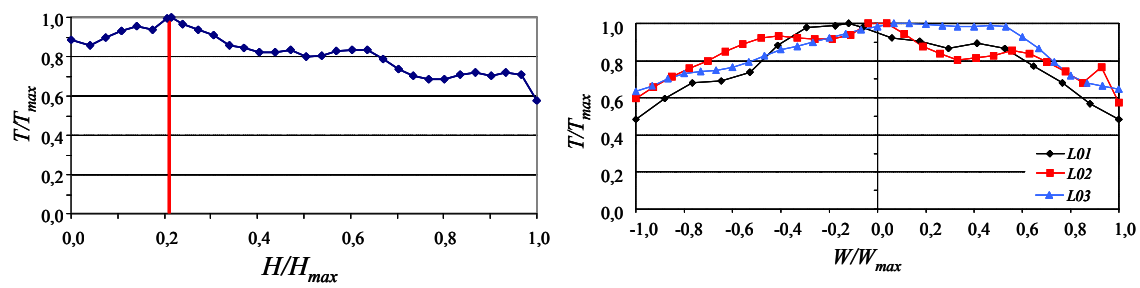

Figure 6: Dimensionless temperature evolution with dimensionless flame height (left) and width (right).

Finally, data registered by the heat flux sensor during the pseudo-stationary phase gave a mean heat flux value of $0.0243 \mathrm{~kW} / \mathrm{m}^{2}$ and $1.7076 \mathrm{~kW} / \mathrm{m}^{2}$ for lab and field tests, respectively. This derived to an emissive power of the flame from the lab test of $10.60 \mathrm{~kW} / \mathrm{m}^{2}$ with a converged value of 0.5 for flame emissivity, and thus validating the hypothesis previously considered. For the field test, an emissive power of the flame of $14 \mathrm{~kW} / \mathrm{m} 2$ was found with a converged emissivity of 0.7 . That gave a mean flame temperature of $748 \mathrm{~K}$.

\section{Conclusions}

Flame length and flame pulse for stationary fires in laboratory and in the open has been determined with an automatic algorithm using a sequence of images extracted from VHS according to flame luminosity. Flame intermittency has been found larger in terms of flame range height and faster in terms of flame pulse frequency in field tests than in smaller laboratory tests.

Flame temperature distribution within the flame has been systematically studied for the stationary lab fire test. A linear evolution has been observed for flame temperature along the axis and across the flame width, giving a maximum flame temperature located at the flame axis, at $1 / 5$ of the total flame height. Combining data from IR imagery and a heat flux transducer, an automated methodology has been designed to obtain emissive power of stationary fires at lab and field scales by which mean flame temperatures and flame emissivity can also be derived. 


\section{References}

[1] Cohen, J.D., Relating flame radiation to home ignition using modeling and experimental crown fires, Canadian Journal of Forest Research, 34, pp. 616-1626, 2004.

[2] Cruz, M.G., Butler, B.W., Alexander, M.E., Forthofer, J.M., Wakimoto, R.M., Predicting the ignition of crown fuels above a spreading surface fire. Part I: model idealization. International Journal of Wildland Fire, 15(1), pp. 47-60, 2006.

[3] Alexander, M.E., Calculating and interpreting forest fire intensities. Canadian Journal of Botany, 60, pp. 349-357, 1982.

[4] Dupuy, J.L., Maréchal, Morvan, D., Fires from a cylindrical forest fuel burner: combustion dynamics and flame properties, Combustion and Flame 135, pp. 65-76, 2003.

[5] Morvan D., Dupuy J.L., Modeling the propagation of a wildfire through a Mediterranean shrub using a multiphase formulation, Combustion and Flame, 138, pp.199-210, 2004.

[6] Anderson, W., Pastor, E., Butler, B., Catchpole, E., Dupuy, J.L., Fernandes, P., Guijarro, M., Mendes.Lopes, J.M., Ventura, J., Evaluating models to estimate flame characteristics for free-burning fires using laboratory and field data, Forest Ecology and Management, 234(1), S77, 2006.

[7] Morandini, F., Balbi, J.H., Rinieri, F. and Marcelli, T., Measurement of pine needles buoyant diffusion flame characteristic length scales using infrared image processing technique, Proc. of the IV International Conference on Forest Fire Research, Coimbra, 18-23 November, 2002.

[8] Mendes-Lopes, J.M.C., Ventura, J.M.P., Amaral, J.M.P., Flame characteristics, temperature-time curves, and rate of spread in fires propagation in a bed of Pinus pinaster needles, International Journal of Wildland Fire 12, pp. 67-84.

[9] Arrue, B., Ollero, A., Common methodologies for collecting data during laboratory fires, Eufirelab EVR1-CT-2002-40028, D-07-11, 2004.

[10] Zukoski, E.E., Cetegen, B.M., Kubota, T, Visible structure of buoyant diffusion flames. In Twentieth Symp. (int.) on Combustion, Pittsburgh, The Combustion Institute, pp. 361-366, 1985.

[11] Ferrero, F., Muñoz, M., Arnaldos, J., Effects of thin-layer boilover on flame geometry and dynamics in large hydrocarbon pool fires, Fuel Processing Technology, 88(3), pp. 227-235, 2007.

[12] Pagni, P.J., Pool fire vortex shedding frequencies. In Some Unanswered Questions in Fluid Mechanics. Ed. L.M. Trefethen and R.L. Panton, vol. 89-WA/FE-5, ASME, 1989. 